\title{
Aberration-corrected STEM/TEM Chemical Analysis and Imaging of Meteoritic Refractory Oxide Assemblages
}

\author{
Tarunika Ramprasad ${ }^{1}$, Prajkta Mane ${ }^{2}$, Thomas J. Zega ${ }^{1,2}$ \\ 1. Department of Materials Science and Engineering, The University of Arizona, Tucson, USA. \\ 2. Lunar and Planetary Laboratory, The University of Arizona, Tucson, USA.
}

Calcium-aluminum rich inclusions (CAIs) are $\mathrm{mm}$ to $\mathrm{cm}$ sized objects found in chondritic meteorites [1]. They are composed of high-temperature mineral phases that were among the first solids to condense in the protosolar nebula [2]. CAIs therefore record within their microstructures information regarding the earliest processes that occurred during the formation of our solar system. Spinels $\left(\mathrm{MgAl}_{2} \mathrm{O}_{4}\right)$ and perovskites $\left(\mathrm{CaTiO}_{3}\right)$ are two important mineral phases in CAIs and are predicted to be some of the earliest solids to condense from the vapor phase [2-3]. CAIs and the mineral assemblages within them are exposed to various environments as they move through the protoplanetary disk before their accretion into the parent body. Analytical techniques with high spatial resolution are required to infer the possible structural and chemical changes resulting from these environmental interactions [4]. Simultaneous structural and chemical analysis capabilities with high spatial resolution make aberration-corrected transmission electron microscopes (TEM) important instruments for the study of CAIs, and the understanding of their formation and early processes in our solar system.

For this study, we analyze spinel-perovskite assemblages in a compact type-A CAI with a core shell structure, from the Northwest Africa (NWA) 5028 chondritic meteorite. A petrographic thin section of NWA 5028 was acquired from the Center for Meteorite Studies, Arizona State University and mapped using a Cameca SX-100 electron microprobe (EMP), located at the Lunar and Planetary Laboratory, University of Arizona (UA/LPL). In the EMP analysis of the selected spinel and perovskite grains, V was observed in solid solution. Two spinel-perovskite assemblages, one from the interior of the inclusion and one from the rim around it, were selected for further analysis in a TEM. This is part of a broader effort to understand the structure of CAI materials from the micrometer down to atomic length scales and to use that information to understand what they tell us about the thermodynamic origins of these materials in the early solar nebula.

The selected areas (section 1 - interior, section 2 - rim) were extracted and thinned to electron transparency using previously described methods [5] with a FEI Helios NanoLAB 660 focused-ionbeam (FIB) scanning electron microscope (SEM) equipped with EDAX energy-dispersive spectroscopy (EDS), located at UA/LPL. The sections were analyzed using the $200 \mathrm{keV}$ spherical-aberrationcorrected Hitachi HF5000 TEM, located in the UA/LPL. The HF5000 is equipped with a Gatan 965 post-column electron energy-loss spectrometer and Oxford Instruments X-max side-entry EDS system equipped with dual SDD detectors proving a large solid angle $(2.0 \mathrm{sr})$ of collection. The TEM was used to acquire EDS maps and selected-area electron-diffraction (SAED) patterns for the various mineral phases present in the FIB sections. EDS maps were acquired using both detectors over 2048 channels, with an energy range of $40 \mathrm{keV}$ and a dwell time of $5 \mu \mathrm{s}$. SAED patterns were acquired with $2048 \times 2048$ resolution ( $2 \mathrm{x}$ binning) using a Gatan OneView CCD camera. Combined information from the EDS maps and SAED patterns enable phase identification.

Section 1 consists of two large grains, one each of spinel $(\sim 6 \mu \mathrm{m})$ and perovskite $(\sim 5.4 \mu \mathrm{m})$ separated by 
a linear interface (Fig. 1). The EDS map of the spinel grain shows that it contains an inclusion, which is composed of a $\mathrm{Ca}-\mathrm{Al}$ silicate grain with a Ti-rich $\mathrm{Ca}-\mathrm{Al}$ silicate rim and an adjoining perovskite grain. Indexing of SAED patterns from these minerals conform to the reference spinel and perovskite structures. Section 2 consists of three perovskite grains $(\sim 3 \mu \mathrm{m}$ each) surrounded by a spinel grain $(\sim 11 \mu \mathrm{m})$ (Fig 2.). The darker contrast of the inclusions within the perovskite grains (similar to the spinel region), combined with the correlative $\mathrm{Mg}$, $\mathrm{Al}$ and $\mathrm{O}$ EDS maps suggests a spinel composition for these domains. SAED patterns from section 2 suggest multiple orientations for the different perovskite grains.

From spatial relationships it can be inferred that the mineral enclosed within another grain formed before the surrounding phase. Based on equilibrium thermodynamics, perovskites are predicted to condense out of the vapor phases at higher temperatures than spinels [2-3]. While the presence of the perovskite grain within the spinel region in section 1 is consistent with equilibrium predictions, the spinel grains within the perovskite regions in section 2 suggest the possibility of deviation from the sequence of minerals predicted to have condensed in the solar protoplanetary disk $[2,3,6]$.

\section{References:}

[1] MacPherson G. J. in "Treatise on Geochemistry. Vol I: Meteorites, Comets and Planets", ed. Davis A.M., (Elsevier, Waltham) p. 201.

[2] Ebel D.S. in "Condensation of rocky material in astrophysical environments. Meteorites and the Early Solar System II", ed. Lauretta D.S. and McSween Jr. H.Y., (University of Arizona Press, Tucson) p. 253.

[3] Lodders K., The Astrophysical Journal 591 (2003), p. 1220.

[4] Simon J.I., et. al., Science 331 (2011), p.1175.

[5] Zega T. J., et. al., Meteoritics \& Planet. Sci. 42 (2007), p. 1373.

[6] We gratefully acknowledge NASA (grants \#NNX12AL47G and \#NNX15AJ22G) and NSF (grants \#1531243 and \#0619599) for funding of instrumentation in the Kuiper Materials Imaging and Characterization Facility at the Lunar and Planetary Laboratory, University of Arizona. Research supported by NASA grant \#NNX15AJ22G.

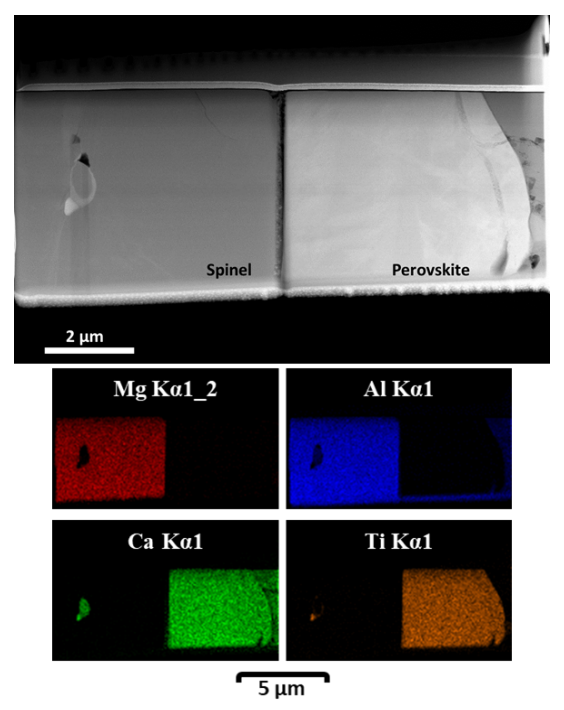

Figure 1. Section 1 data:

HAADF Image (top), EDS maps (bottom)

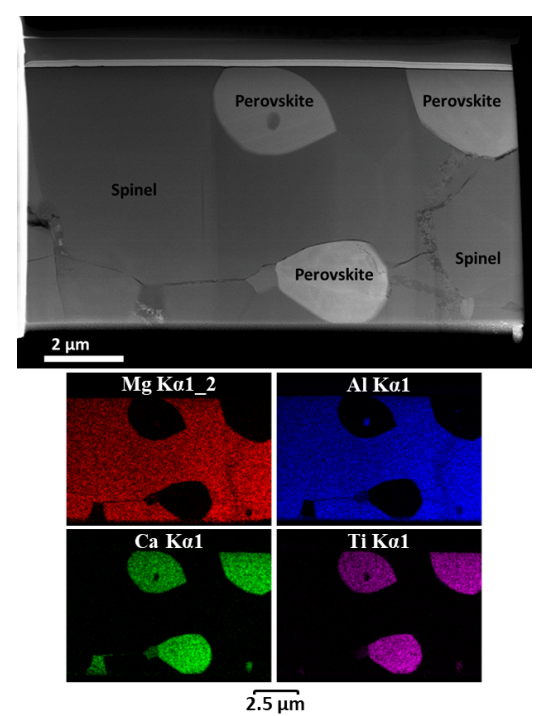

Figure 2. Section 2 data:

HAADF Image (top), EDS maps (bottom) 